\title{
ANALYSIS OF OSCILLATORY CONTROL SYSTEMS
}

\author{
Sonia Martínez ${ }^{*, 1}$ Jorge Cortés ${ }^{*, 2}$ Francesco Bullo ${ }^{* *, 3}$ \\ * Instituto de Matemáticas y Física Fundamental, Consejo \\ Superior de Investigaciones Cientificas, Serrano 123, Madrid, \\ 28006, Spain, Tel: +1-34-915616800 (extensions 1110 and 1114), \\ Fax: +1-34-915854894, \\ Email: $\{$ s.martinez,j.cortes\}@imaff.cfmac.csic.es \\ ** Coordinated Science Laboratory, University of Illinois at \\ Urbana-Champaign, 1308 W. Main St., Urbana, IL 61801, \\ United States, Tel: +1-217-3330656, Fax: +1-217-2441653, \\ Email: bullo@uiuc.edu
}

\begin{abstract}
:
This paper presents analysis results for control systems subject to oscillatory inputs, i.e., inputs of large amplitude and high frequency. The key results are a series expansion characterizing the averaged system and various Lie-algebraic conditions that guarantee the series can be summed. Some illustrative example systems provide insight into the results; control design applications are discussed in a companion paper.
\end{abstract}

Keywords: oscillatory control, averaging, geometric methods

\section{INTRODUCTION}

The study of oscillations in nonlinear differential equations is a classic and widespread research topic. Related research areas include nonlinear dynamical systems (Hale 1992), nonlinear and geometric control (Fliess et al. 1995), analysis of animal locomotion (Golubitsky et al. 1999), design of robotic locomotion and manipulation devices (Hirose 1993), analysis of switching circuit models and power conversion circuits (Sanders et al. 1991), control of quantum dynamics (Warren et al. 1993) and chemical reactions (Paramonov 1993), and so forth. Furthermore, averaging analysis seems well suited to tackle novel applications in the field of micro-electro mechanical systems (Baillieul and Weibel 1998) and vibrational control is being investigated within the context of active control of fluids and separation control (Seifert and Pack 1999).

\footnotetext{
1 Partially supported by a FPI grant from Spanish MCYT.

2 Partially supported by a FPU grant from Spanish MEC.

3 Partially supported by NSF grant CMS-0100162.
}

Here we present averaging analysis for systems described by a differential equation of the form

$$
\dot{x}=f(t, x)+\frac{1}{\epsilon} g\left(\frac{t}{\epsilon}, t, x\right),
$$

where the vector field $g$ is periodic in its first argument, $\epsilon$ is a small positive parameter, and both vector fields $f$ and $g$ are analytic in $x$. We provide a rigorous and general framework that allows to obtain a coordinate-free expression of the averaged system and a series expansion representation for it which is appropriate for control design tasks such as point stabilization and trajectory planning in underactuated systems. Due to space limitations we do not present these applications here, but refer the reader to a forthcoming publication (Martínez et al. 2003).

This work has connections with classic averaging theory (Sanders and Verhulst 1985), as well as with numerous ongoing research efforts. Our analysis complements the study of differential equations subject to periodic high frequency, high amplitude forcing terms; see (Kurzweil and Jarnik 1988, Liu and Sussmann 1999). Another set of 
related results deals with the analysis of high frequency vibrations in mechanical systems (Kapitsa 1965, Baillieul 1993, Baillieul 1995, Levi 1999), and more generally averaging analysis in locomotion, rectification and other physical phenomenon where non-commuting vector fields play a role; see (Brockett 1989, Ostrowski and Burdick 1998).

The contribution of this paper is a general averaging analysis in a coordinate-free differential geometric setting. The first step in the averaging analysis is the computation of the averaged system based on a generalized variation of constants formula and on the notion of pull-back vector field. Next, we provide a more explicit representation of the averaged system as an infinite sum of Lie brackets of the input vector fields with the drift and iterated integrals of the open-loop controls. Finally, we present various examples systems including bilinear, Hamiltonian, and second order systems, and collect sufficient conditions that guarantee the series expansion for the averaged system is summable.

The paper is organized as follows. We introduce some preliminary concepts in Section 2. Section 3 presents the main averaging analysis in a coordinate-free differential geometric setting. Section 4 treats various example systems and Section 5 presents some conclusions.

\section{PRELIMINARIES AND NOTATION}

This section contains some basic definitions and results on iterated integrals of scalar functions and on differential geometry.

\subsection{Iterated integrals and their averages}

Let $\mathbb{N}$ be the set of natural numbers and $\mathbb{R}_{+}=$ $[0,+\infty)$. Given a bounded function $u: \mathbb{R}_{+} \rightarrow \mathbb{R}$, define its iterated integrals $\left\{U_{k}: \mathbb{R}_{+} \rightarrow \mathbb{R}, k \in \mathbb{N}\right\}$,

$U_{k}(t)=\int_{0}^{t} u\left(t_{k}\right) \int_{0}^{t_{k}} u\left(t_{k-1}\right) \ldots \int_{0}^{t_{2}} u\left(t_{1}\right) d t_{1} \ldots d t_{k}$.

Lemma 1. Let $u$ be a bounded function. Its iterated integrals satisfy

$$
U_{k}(t)=\frac{1}{k !}\left(\int_{0}^{t} u(\tau) d \tau\right)^{k},
$$

and are $T$-periodic functions if and only if $u$ is $T$-periodic and zero-mean, i.e., $\int_{0}^{T} u(\tau) d \tau=0$.

Proof: The result is easily seen true for $k=1$. For $k>1$, we have

$$
\frac{d}{d t} U_{k}(t)=\frac{u(t)}{(k-1) !}\left(\int_{0}^{t} u(\tau) d \tau\right)^{k-1}=U_{k-1}(t) u(t),
$$

which implies that the $U_{k}$ satisfy

$$
U_{k}(t)=\int_{0}^{t} U_{k-1}(\tau) u(\tau) d \tau
$$

Since this equation is equivalent to the definition of iterated integral, equation (1) is proven. The second part of the result is straightforward.

Given a $T$-periodic function $V(t)$, let us denote

$$
\bar{V}=\frac{1}{T} \int_{0}^{T} V(t) d t .
$$

If $u$ is $T$-periodic and zero-mean, the sequence of its averages $\left\{\bar{U}_{k} \in \mathbb{R}, k \in \mathbb{N}\right\}$ is defined as

$\bar{U}_{k}=\frac{1}{T} \int_{0}^{T} U_{k}(t) d t=\frac{1}{k ! T} \int_{0}^{T}\left(\int_{0}^{t} u(\tau) d \tau\right)^{k} d t$.

\subsection{Elements of differential geometry}

Let $x, x_{0} \in \mathbb{R}^{n}, t \in \mathbb{R}_{+}$, and let the parameter $\epsilon$ vary in the range $\left(0, \epsilon_{0}\right]$ with $\epsilon_{0} \ll 1$. Let $f, g: \mathbb{R}_{+} \times \mathbb{R}^{n} \rightarrow \mathbb{R}^{n}$ be smooth time-varying vector fields. Define their Lie bracket according to

$$
[g, f]=\frac{\partial f(t, x)}{\partial x} g(t, x)-\frac{\partial g(t, x)}{\partial x} f(t, x) .
$$

We will use the notation $\operatorname{ad}_{g}^{0} f=f, \operatorname{ad}_{g} f=[g, f]$ and $\operatorname{ad}_{g}^{k} f=\operatorname{ad}_{g}^{k-1}[g, f]$. Given a diffeomorphism $\phi$ and a vector field $f$, the pull-back of $g$ along $\phi$, denoted by $\phi^{*} f$, is the vector field

$$
\left(\phi^{*} f\right)(x)=\left(\frac{\partial \phi^{-1}}{\partial x} \circ f \circ \phi\right)(x) .
$$

A useful diffeomorphism is the flow map $x(t)=$ $\Phi_{0, T}^{g}\left(x_{0}\right)$ describing the solution at time $T$ to the initial value problem $\dot{x}=g(t, x), \quad x(0)=x_{0}$. Let $T>0$ be small enough so that the flow map $\Phi_{0, T}^{g}$ is a local diffeomorphism in a neighborhood of $x_{0}$.

Lemma 2. (Variation of constants formula). Consider the initial value problem $\dot{x}=f(t, x)+g(t, x)$, $x(0)=x_{0}$. The final value $x(T)=\Phi_{0, T}^{f+g}\left(x_{0}\right)$ can be written as

$$
\begin{aligned}
x(T) & =\Phi_{0, T}^{g}(z(T)), \\
\dot{z}(t) & =\left(\left(\Phi_{0, t}^{g}\right)^{*} f\right)(z), \quad z(0)=x_{0} .
\end{aligned}
$$

Proof: See (Agrachev and Gamkrelidze 1978).

\section{COORDINATE-FREE AVERAGING UNDER OSCILLATORY CONTROLS}

We study averaging under oscillatory controls, borrowing standard ideas from (Sanders and 
Verhulst 1985), and introducing some differential geometric aspects from (Agrachev and Gamkrelidze 1978, Hermes 1991).

Let $x:[0, T] \rightarrow \mathbb{R}^{n}$ be the solution to

$$
\frac{d x}{d t}=f(t, x)+\frac{1}{\epsilon} g\left(\frac{t}{\epsilon}, t, x\right), \quad x(0)=x_{0} .
$$

Enlarge the state space by considering $x^{\prime}=(t, x)$, denote by $\tau=t / \epsilon$ the fast time scale, and rewrite (3) as

$$
\frac{d x^{\prime}}{d \tau}=\epsilon f^{\prime}\left(x^{\prime}\right)+g^{\prime}\left(\tau, x^{\prime}\right), \quad x_{0}^{\prime}=\left(0, x_{0}\right),
$$

where the vector fields $f^{\prime}$ and $g^{\prime}$ are defined according to

$$
f^{\prime}\left(x^{\prime}\right)=(1, f(t, x)), \quad g^{\prime}\left(\tau, x^{\prime}\right)=(0, g(\tau, t, x)) .
$$

In the extended space, $\tau$ is the independent variable and $(t, x)$ are dependent variables. We shall write the flow of $g^{\prime}$ as

$$
\Phi_{0, \tau}^{g^{\prime}\left(\tau, x^{\prime}\right)}(t, x)=\left(t, \Phi_{0, \tau}^{g(\tau, t, x)}(x)\right),
$$

and define the pull-back vector field $F^{\prime}$ as

$$
F^{\prime}\left(\tau, x^{\prime}\right)=\left(\left(\Phi_{0, \tau}^{g^{\prime}\left(\tau, x^{\prime}\right)}\right)^{*} f^{\prime}\right)\left(x^{\prime}\right) .
$$

Note that $F^{\prime}$ is of the form

$$
F^{\prime}\left(\tau, x^{\prime}\right)=\left(1, F\left(\tau, x^{\prime}\right)\right) .
$$

Lemma 3. Assume that the vector fields in $\left\{g_{\tau}\right.$, $\tau \in[0, T]\}$ commute and satisfy $g(\tau+T, t, x)=$ $g(\tau, t, x)$, and $\int_{0}^{T} g(\tau, t, x) d \tau=0$. Then, the flow $\Phi_{0, \tau}^{g^{\prime}\left(\tau, x^{\prime}\right)}$ and the vector field $F^{\prime}$ are $T$-periodic.

Proof: The assumptions on the family $\left\{g_{\tau}, \tau \in\right.$ $[0, T]\}$ are automatically verified by $\left\{g_{\tau}^{\prime}, \tau \in\right.$ $[0, T]\}$. Let $x^{\prime}(t)$ be the solution to the initial value problem

$$
\dot{x}^{\prime}(\tau)=g^{\prime}\left(\tau, x^{\prime}\right), \quad x^{\prime}(0)=x_{0}^{\prime} .
$$

Note that $x^{\prime}(\tau)=\Phi_{0, \tau}^{g^{\prime}\left(\tau, x^{\prime}\right)}\left(x_{0}^{\prime}\right)$. Let $X^{\prime}(\tau)=$ $x^{\prime}(\tau+T)$. Then

$\dot{X}^{\prime}(\tau)=g^{\prime}\left(\tau+T, X^{\prime}\right)=g^{\prime}\left(\tau, X^{\prime}\right), X^{\prime}(0)=x^{\prime}(T)$.

Consequently, $X^{\prime}(\tau)=x^{\prime}(\tau)$ iff $x^{\prime}(T)=x^{\prime}(0)$. To prove the latter statement we introduce the Volterra series (Agrachev and Gamkrelidze 1978, Isidori 1995). The flow of (7) is formally represented by the expansion

$$
\begin{aligned}
& x^{\prime}(\tau) \equiv \operatorname{Id}\left(x_{0}^{\prime}\right)+ \\
& \sum_{k=1}^{+\infty} \int_{0}^{\tau} d s_{1} \ldots \int_{0}^{s_{k-1}} d s_{k}\left(g^{\prime}\left(s_{k}, x_{0}^{\prime}\right) \circ \cdots \circ g^{\prime}\left(s_{1}, x_{0}^{\prime}\right)\right) \\
& =x_{0}^{\prime}+\int_{0}^{\tau} g^{\prime}\left(s, x_{0}^{\prime}\right) d s+ \\
& \quad \int_{0}^{\tau} \int_{0}^{s_{1}}\left(g^{\prime}\left(s_{2}, x_{0}^{\prime}\right) \circ g^{\prime}\left(s_{1}, x_{0}^{\prime}\right)\right) d s_{2} d s_{1}+\ldots
\end{aligned}
$$

where the vector fields $g^{\prime}$ are interpreted as derivations of $C^{\infty}\left(\mathbb{R}^{n+1}\right)$. Now, using integration by parts and the fact that the vector fields commute,

$$
\begin{aligned}
\int_{0}^{\tau} \int_{0}^{s_{1}}\left(g^{\prime}\left(s_{2}, x_{0}^{\prime}\right) \circ g^{\prime}\right. & \left.\left(s_{1}, x_{0}^{\prime}\right)\right) d s_{2} d s_{1} \\
& =\frac{1}{2}\left(\int_{0}^{\tau} g^{\prime}\left(s, x_{0}^{\prime}\right) d s\right)^{2} .
\end{aligned}
$$

By induction, one can show that

$$
\begin{array}{rl}
\int_{0}^{\tau} d s_{1} \ldots \int_{0}^{s_{k-1}} & d s_{k}\left(g^{\prime}\left(s_{k}, x_{0}^{\prime}\right) \circ \cdots \circ g^{\prime}\left(s_{1}, x_{0}^{\prime}\right)\right) \\
= & \frac{1}{k !}\left(\int_{0}^{\tau} g^{\prime}\left(s, x_{0}^{\prime}\right) d s\right)^{k} \cdot \quad(9)
\end{array}
$$

Since by hypothesis $g^{\prime}\left(\tau, x^{\prime}\right)$ is zero-mean, we conclude from (8) that $x^{\prime}(T)=x^{\prime}(0)$.

Given the result in the lemma, we define the averaged vector field $\overline{F^{\prime}}$ as

$$
\overline{F^{\prime}}\left(x^{\prime}\right)=\frac{1}{T} \int_{0}^{T} F^{\prime}\left(\tau, x^{\prime}\right) d \tau .
$$

It is straightforward to see that $\overline{F^{\prime}}\left(x^{\prime}\right)=\left(1, \bar{F}\left(x^{\prime}\right)\right)$. Finally, let $y, z:[0, T] \rightarrow \mathbb{R}^{n}$ be solutions to the initial value problems

$$
\begin{aligned}
& \frac{d z}{d t}=F\left(\frac{t}{\epsilon}, t, z\right), \quad z(0)=x_{0}, \\
& \frac{d y}{d t}=\bar{F}(t, y), \quad y(0)=x_{0} .
\end{aligned}
$$

Theorem 4. (Coordinate-free averaging). Assume that the vector fields in $\left\{g_{\tau}, \tau \in[0, T]\right\}$ commute and that they are $T$-periodic and zero mean in their first argument. For $t \in \mathbb{R}_{+}$, we have

$$
x(t)=\Phi_{0, t / \epsilon}^{g(\tau, t, x)}(z(t)),
$$

and, as $\epsilon \rightarrow 0$ on the time scale 1 , we have

$$
z(t)-y(t)=O(\epsilon) .
$$

Additionally, assume both $f$ and $g$ do not depend explicitly on the slow time scale $t$, i.e. $f=f(x)$ and $g=g(t / \epsilon, x)$. If the origin is a hyperbolically stable critical point for $\bar{F}=\bar{F}(x)$, then $z(t)-$ $y(t)=O(\epsilon)$ as $\epsilon \rightarrow 0$ for all $t \in \mathbb{R}_{+}$and the differential equation (10) possesses a unique periodic orbit which is hyperbolically stable and belongs to an $O(\epsilon)$ neighborhood of the origin.

Proof: Applying the variation of constants formula to (4), we get

$$
\begin{array}{ll}
\frac{d x^{\prime}}{d \tau}=g^{\prime}\left(\tau, x^{\prime}\right), & x^{\prime}(0)=z^{\prime}(\tau), \\
\frac{d z^{\prime}}{d \tau}=\epsilon F^{\prime}\left(\tau, z^{\prime}\right), & z^{\prime}(0)=x_{0}^{\prime} .
\end{array}
$$

Averaging this last system in $z^{\prime}$, we obtain

$$
\frac{d y^{\prime}}{d \tau}=\epsilon \overline{F^{\prime}}\left(y^{\prime}\right), \quad y^{\prime}(0)=x_{0}^{\prime} .
$$


By the theorem of first order averaging (Sanders and Verhulst 1985), we know that $z^{\prime}(\tau)-y^{\prime}(\tau)=$ $O(\epsilon)$ over the time scale $\tau=1 / \epsilon$. Now, if we write $y^{\prime}=(v, y)$, we get from the previous equation that $v=t$ and, changing the time scale back to $t=\epsilon \tau$,

$$
\frac{d y}{d t}=\bar{F}(t, y), \quad y(0)=x_{0},
$$

which is the definition of equation (11). Putting $z^{\prime}=(u, z)$, we also deduce that $u=t$ and $z(t)-$ $y(t)=O(\epsilon)$ over the time scale 1 . In addition, we recover equation (10)

$$
\frac{d z}{d t}=F\left(\frac{t}{\epsilon}, t, z\right), \quad z(0)=x_{0} .
$$

Finally, we get from (12) that

$$
x(t)=\Phi_{0, t / \epsilon}^{g(\tau, t, x)}(z(t)) .
$$

In case $f=f(x)$ and $g=g(t / \epsilon, x)$, if the origin is a hyperbolically stable critical point for $\bar{F}$, then the second result follows from the theorem of first order averaging (Sanders and Verhulst 1985).

Next, we refine the approximation result as follows. Let $\lfloor s\rfloor$ be the greatest integer less than or equal to $s \in \mathbb{R}$, and let $(t / \epsilon \bmod T)$ denote the time instant $t / \epsilon-\lfloor t /(\epsilon T)\rfloor T$.

Proposition 5. Under the hypothesis of Theo$\operatorname{rem} 4$, if $T=O(1)$, then $x(t)=\Phi_{0,(t / \epsilon \bmod T)}^{g(\tau, t, x)}(y(t))$ $+O(\epsilon)$, on the time scale 1 .

Proof: The proof exploits Lemma 3 and the Volterra series expansion for the flow $\Phi_{0, t / \epsilon}^{g(s, t, x)}$ (see (Martínez et al. 2003)).

\subsection{Series expansions for the averaged system}

In this section, we develop a series expansions for the averaged system in the single input case. For the multiple input case as well as the convergence properties of the series, we refer to (Martínez et al. 2003).

Assume that the input vector field in equation (3) is of the form $g(t / \epsilon, t, x)=u(t / \epsilon, t) g(x)$, where $u$ is a bounded function. In other words, equation (3) reads

$$
\frac{d x}{d t}=f(t, x)+\frac{1}{\epsilon} u\left(\frac{t}{\epsilon}, t\right) g(x), \quad x(0)=x_{0} .
$$

Accordingly, we shall consider the iterated integrals $U_{k}(\tau, t)$ and their averages $\bar{U}_{k}(t)$ with respect to the first variable of the input $u(\tau, t)$.

Theorem 6. (Single input system). Let $(\tau, t) \mapsto$ $u(\tau, t)$ be a bounded function, $T$-periodic and zero-mean in $\tau$, continuously differentiable in $t$.
The vector field $F$ defined in equation (6) satisfies the formal expansion

$$
\begin{aligned}
& F(\tau, t, x)=f(t, x) \\
& +\sum_{k=1}^{+\infty} U_{k}(\tau, t) \operatorname{ad}_{g}^{k} f(t, x)-\frac{\partial U_{1}}{\partial t}(\tau, t) g(x)
\end{aligned}
$$

and its average $\bar{F}$ satisfies

$$
\begin{aligned}
& \bar{F}(t, x)=f(t, x) \\
& \quad+\sum_{k=1}^{+\infty} \bar{U}_{k}(t) \operatorname{ad}_{g}^{k} f(t, x)-\frac{d \bar{U}_{1}}{d t}(t) g(x) .
\end{aligned}
$$

Proof: We compute $F^{\prime}$ as in equation (5), where we let $f^{\prime}=f^{\prime}\left(x^{\prime}\right)$ be $\tau$-invariant and $g^{\prime}=g^{\prime}\left(\tau, x^{\prime}\right)$ be $\tau$-varying. The following statement is proved in (Abraham et al. 1988, Theorem 4.2.31)

$\frac{d}{d \tau}\left(\left(\Phi_{0, \tau}^{g^{\prime}}\right)^{*} f^{\prime}\right)\left(\tau, x^{\prime}\right)=\left(\Phi_{0, \tau}^{g^{\prime}}\right)^{*}\left[g^{\prime}\left(\tau, x^{\prime}\right), f^{\prime}\left(x^{\prime}\right)\right]$.

At fixed $x^{\prime} \in \mathbb{R}^{n+1}$, we integrate the previous equation from time 0 to $\tau$ to obtain

$$
\begin{aligned}
& \left(\left(\Phi_{0, \tau}^{g^{\prime}}\right)^{*} f^{\prime}\right)\left(\tau, x^{\prime}\right)= \\
& f^{\prime}\left(x^{\prime}\right)+\int_{0}^{\tau}\left(\Phi_{0, s}^{g^{\prime}}\right)^{*}\left[g^{\prime}\left(s, x^{\prime}\right), f^{\prime}\left(x^{\prime}\right)\right] d s .
\end{aligned}
$$

Iteratively applying the previous equality, we get

$$
\begin{gathered}
\left(\left(\Phi_{0, \tau}^{g^{\prime}}\right)^{*} f^{\prime}\right)\left(\tau, x^{\prime}\right)=f^{\prime}\left(x^{\prime}\right)+\sum_{k=1}^{+\infty} \int_{0}^{\tau} \ldots \int_{0}^{s_{k-1}} \\
\left(\operatorname{ad}_{g^{\prime}\left(s_{k}, x^{\prime}\right)} \ldots \operatorname{ad}_{g^{\prime}\left(s_{1}, x^{\prime}\right)} f^{\prime}\left(x^{\prime}\right)\right) d s_{k} \ldots d s_{1}
\end{gathered}
$$

Now, it can be proven by induction that

$$
\begin{aligned}
& \operatorname{ad}_{g^{\prime}\left(s_{1}, t, x\right)} f^{\prime}= \\
& \left(0, u\left(s_{1}, t\right) \operatorname{ad}_{g(x)} f-\frac{\partial u}{\partial t}\left(s_{1}, t\right) g(x)\right), \\
& \operatorname{ad}_{g^{\prime}\left(s_{k}, x^{\prime}\right)} \ldots \operatorname{ad}_{g^{\prime}\left(s_{1}, x^{\prime}\right)} f^{\prime}= \\
& \left(0, u\left(s_{k}, t\right) \ldots u\left(s_{1}, t\right) \operatorname{ad}_{g(x)}^{k} f\right) .
\end{aligned}
$$

with $k \geq 2$. The result now follows.

\section{EXAMPLE SYSTEMS}

Here we investigate classes of differential equations for which the series expansions in Section 3.1 assume a particular structure. By doing so, we recover and extend a variety of earlier results on bilinear, polynomial and second order systems.

Before proceeding, we summarize the averaging procedure from Theorem 4 and Proposition 5 as follows,

$$
\begin{aligned}
x(t) & =\Phi_{0,(t / \epsilon \bmod T)}^{g(t, x)}(y(t))+O(\epsilon), \\
\dot{y} & =\bar{F}(y), y(0)=x_{0} .
\end{aligned}
$$

Accordingly, for each example we shall compute $\Phi_{0,(t / \epsilon \bmod T)}^{g(t, x)}$ and $\bar{F}$. 


\subsection{Homogeneous systems}

Let $f$ be a vector field on $\mathbb{R}^{n}$. We say that $f$ is homogeneous of degree $i$ if each of its components with respect to the usual basis of $\mathbb{R}^{n}$ is a homogeneous function of degree $i$. The set of homogeneous vector fields of degree $i$ is denoted by $\mathcal{H}_{i}$. By convention, $\mathcal{H}_{i}=\{0\}$, for $i \leq-1$. If $f \in \mathcal{H}_{i}$ and $g \in \mathcal{H}_{j}$, then $[f, g] \in \mathcal{H}_{i+j-1}$.

The relevant quantities from Theorems 4 and 6 for the cases $(\operatorname{deg} f, \operatorname{deg} g)=(0,0),(0,1),(1,0)$ are

$$
\begin{aligned}
& \text { Case }(0,0): \dot{x}=a+\frac{1}{\epsilon} u\left(\frac{t}{\epsilon}\right) b, \quad x(0)=x_{0}, \\
& \Phi_{0,(t / \epsilon \bmod T)}^{u(t) b}\left(x_{0}\right)=b \int_{0}^{(t / \epsilon \bmod T)} u(\tau) d \tau+x_{0}, \\
& \bar{F}=a .
\end{aligned}
$$$$
\text { Case }(0,1): \dot{x}=a+\frac{1}{\epsilon} u\left(\frac{t}{\epsilon}\right) B x, \quad x(0)=x_{0},
$$$$
\Phi_{0,(t / \epsilon \bmod T)}^{u(t) B x}\left(x_{0}\right)=\mathrm{e}^{B \int_{0}^{(t / \epsilon \bmod T)} u(\tau) d \tau} x_{0},
$$$$
\bar{F}=a+\sum_{k=1}^{+\infty} \bar{U}_{k}(-B)^{k} a \text {. }
$$

Case (1,0): $\dot{x}=A x+\frac{1}{\epsilon} u\left(\frac{t}{\epsilon}\right) b, \quad x(0)=x_{0}$,

$$
\Phi_{0,(t / \epsilon \bmod T)}^{u(t) b}\left(x_{0}\right)=b \int_{0}^{(t / \epsilon \bmod T)} u(\tau) d \tau+x_{0},
$$

$\bar{F}=A x+\bar{U}_{1} A b$.

\subsection{Bilinear systems}

We refer the reader to (Isidori 1995, Section 2.4) for a treatment on bilinear systems. Consider

$$
\dot{x}=A x+\frac{1}{\epsilon} u\left(\frac{t}{\epsilon}\right) B x, \quad x(0)=x_{0} .
$$

Lie brackets between linear vector fields are expressed in terms of matrix commutators

$\operatorname{ad}_{B x} A x=\left(\operatorname{ad}_{B} A\right) x$, where $\operatorname{ad}_{B} A=A B-B A$.

One can compute,

$$
\begin{aligned}
& \Phi_{0,(t / \epsilon \bmod T)}^{g u(t)}\left(x_{0}\right)=\mathrm{e}^{B \int_{0}^{(t / \epsilon \bmod T)} u(\tau) d \tau} x_{0}, \\
& \bar{F}(x)=\left(A+\sum_{k=1}^{+\infty} \bar{U}_{k} \operatorname{ad}_{B}^{k} A\right) x .
\end{aligned}
$$

\subsection{Polynomial systems}

Consider the system

$$
\dot{x}=f(x)+\frac{1}{\epsilon} u\left(\frac{t}{\epsilon}\right) g, \quad x(0)=x_{0},
$$

where the components of $f$ are polynomials in $x$ of degree at most $M$, and $g(x)=g$ is constant.
The degree of $\operatorname{ad}_{g}^{k} f$ is $M-k$, and only the first $M$ Lie brackets are non-vanishing. Accordingly,

$$
\begin{aligned}
& \Phi_{0,(t / \epsilon \bmod T)}^{g u(t)}\left(x_{0}\right)=x_{0}+\left(\int_{0}^{(t / \epsilon \bmod T)} u(\tau) d \tau\right) g, \\
& \bar{F}(x)=f(x)+\sum_{k=1}^{M} \bar{U}_{k} \frac{\partial^{k} f}{\partial x^{k}}(\underbrace{g, \ldots, g}_{k \text { times }})(x) .
\end{aligned}
$$

Note that $\bar{F}$ is a finite sum of polynomial vector fields.

\subsection{Second order systems}

We next focus on control systems described by second order differential equations. This setting is representative of interesting examples. Consider the second order system on $\mathbb{R}^{n}$

$$
\ddot{x}=\frac{1}{\epsilon} u\left(\frac{t}{\epsilon}\right) g(x) .
$$

To write the equation in the standard (first order) form (13), define the vector fields on $\mathbb{R}^{2 n}$

$$
f(x, \dot{x})=\left[\begin{array}{c}
\dot{x} \\
0
\end{array}\right], \quad g(x, \dot{x})^{\text {lift }}=\left[\begin{array}{c}
0 \\
g(x)
\end{array}\right],
$$

and compute the relevant Lie brackets as

$$
\begin{array}{r}
\operatorname{ad}_{g^{\text {lift }}} f=\left[\begin{array}{c}
g \\
-\frac{\partial g}{\partial x} \dot{x}
\end{array}\right], \operatorname{ad}_{g^{\text {lift }} f}^{2}=-\langle g: g\rangle^{\text {lift }}, \\
\operatorname{ad}_{g^{\text {lift }}}^{k} f=0, k>2,
\end{array}
$$

where we define the operation of symmetric prod$u c t$ between vector fields $g_{a}, g_{b}$ on $\mathbb{R}^{n}$ as

$$
\left\langle g_{a}: g_{b}\right\rangle=\frac{\partial g_{a}}{\partial x} g_{b}+\frac{\partial g_{b}}{\partial x} g_{a}
$$

The relevant quantities from Theorem 4 and Theorem 6 are

$$
\begin{gathered}
\Phi_{0, t}^{g(x, \dot{x})^{\text {lift }} u(t)}\left(\begin{array}{c}
x_{0} \\
\dot{x}_{0}
\end{array}\right)=\left(\begin{array}{c}
x_{0} \\
\dot{x}_{0}
\end{array}\right)+\left[\left(\int_{0}^{t} u(\tau) d \tau\right) g\left(x_{0}\right)\right], \\
\bar{F}=f+\bar{U}_{1} \operatorname{ad}_{g^{\text {lift }}} f+\bar{U}_{2} \operatorname{ad}_{g^{\text {lift }}}^{2} f \\
=\left[\begin{array}{c}
\dot{x} \\
0
\end{array}\right]+\bar{U}_{1}\left[\begin{array}{c}
g \\
-\frac{\partial g}{\partial x} \dot{x}
\end{array}\right]-\bar{U}_{2}\left[\begin{array}{c}
0 \\
\langle g: g\rangle
\end{array}\right],
\end{gathered}
$$

so that, using the variables $\left(y_{1}, y_{2}\right)$ for the averaged system, we write

$$
\begin{aligned}
& \dot{y}_{1}=y_{2}+\bar{U}_{1} g\left(y_{1}\right) \\
& \dot{y}_{2}=-\bar{U}_{1} \frac{\partial g}{\partial x}\left(y_{1}\right) y_{2}-\bar{U}_{2}\langle g: g\rangle\left(y_{1}\right)
\end{aligned}
$$

with initial conditions $\left(y_{1}(0), y_{2}(0)\right)=(x(0), \dot{x}(0))$. It is instructive to compute the second time derivative of $y_{1}$, and rewrite the averaged system as a second order equation. Some straightforward simplifications lead to

$$
\ddot{y}_{1}=\left(\frac{1}{2} \bar{U}_{1}^{2}-\bar{U}_{2}\right)\langle g: g\rangle\left(y_{1}\right),
$$


with initial conditions $\left(y_{1}(0), \dot{y}_{1}(0)\right)=(x(0), \dot{x}(0)+$ $\left.\bar{U}_{1} g(x(0))\right)$. In summary, we have

$x(t)=y_{1}(t)+O(\epsilon)$

$\dot{x}(t)=\dot{y}_{1}(t)+g\left(y_{1}(t)\right)\left(\int_{0}^{(t / \epsilon \bmod T)} u(s) d s-\bar{U}_{1}\right)+O(\epsilon)$.

Remark 4.1. Analogues to the result in equation (20) and their physical meaning have been long studied; e.g., see (Kapitsa 1965, Baillieul 1993, Levi 1999). In particular, if $g$ is a potential field, $g=\partial V / \partial x$, then $\langle g: g\rangle=\partial W / \partial x$, where $W=(\partial V / \partial x)^{2}$ is the classical Kapitsa's potential (Kapitsa 1965) (also called the averaged potential). It is easy to see that every isolated critical point of $V$ is a minimum of $W$. Using Hölder inequality, we obtain $\frac{1}{2} \bar{U}_{1}^{2}-\bar{U}_{2}<0$ and we conclude that every isolated equilibrium point of the original system is a Lyapunov-stable equilibrium point for the averaged system (Levi 1999).

\section{CONCLUSIONS}

We have presented a coordinate-free averaging analysis for a class of control systems subject to oscillatory inputs. The companion paper (Martínez et al. 2003) developes design tools for stabilization and trajectory tracking in certain classes of nonlinear systems. Future directions of research include deriving extensions of these results to the case of higher-order averaging, distributed parameter systems, time-delayed systems, and systems with resonances.

\section{REFERENCES}

Abraham, R., J. E. Marsden and T. S. Ratiu (1988). Manifolds, Tensor Analysis, and Applications. Vol. 75 of Applied Mathematical Sciences. 2 ed.. Springer Verlag. New York.

Agrachev, A. A. and R. V. Gamkrelidze (1978). The exponential representation of flows and the chronological calculus. Math. USSR Sbornik 35(6), 727-785.

Baillieul, J. (1993). Stable average motions of mechanical systems subject to periodic forcing. In: Dynamics and Control of Mechanical Systems: The Falling Cat and Related Problems (M. J. Enos, Ed.). Vol. 1. pp. 1-23. Field Institute Communications.

Baillieul, J. (1995). Energy methods for stability of bilinear systems with oscillatory inputs. International Journal on Robust and Nonlinear Control 5(4), 285-301.

Baillieul, J. and S. Weibel (1998). Scale dependence in the oscillatory control of micromechanisms. In: IEEE Conf. on Decision and Control. Tampa, FL. pp. 3058-3063.

Brockett, R. W. (1989). On the rectification of vibratory motion. Sensors \& Actuators 20(12), 91-6.
Fliess, M., J. Lévine, P. Martin and P. Rouchon (1995). Flatness and defect of non-linear systems: Introductory theory and examples. International Journal of Control 61(6), 13271361.

Golubitsky, M., I. Stewart, P.-L. Buono and J. J. Collins (1999). Symmetry in locomotor central pattern generators and animal gaits. $\mathrm{Na}$ ture 401(6754), 693-5.

Hale, J. K. (1992). Oscillations in Nonlinear Systems. Dover Publications, Inc.. New York.

Hermes, H. (1991). Nilpotent and high-order approximations of vector field systems. SIAM Review 33(2), 238-264.

Hirose, S. (1993). Biologically inspired robots: snake-like locomotors and manipulators. Oxford University Press. Oxford, UK.

Isidori, A. (1995). Nonlinear Control Systems. 3 ed.. Springer Verlag. New York.

Kapitsa, P. L. (1965). Dynamical stability of a pendulum when its point of suspension vibrates. In: Collected Papers by P. L. Kapitsa. Vol. II. Pergamon. London. pp. 714-725.

Kurzweil, J. and J. Jarnik (1988). Iterated Lie brackets in limit processes in ordinary differential equations. Results in Mathematics 14, 125-137.

Levi, M. (1999). Geometry and physics of averaging with applications. Physica D 132(12), 150-64.

Liu, W. and H. J. Sussmann (1999). Continuous dependence with respect to the input of trajectories of control-affine systems. SIAM Journal on Control and Optimization 37(3), 777-803.

Martínez, S., J. Cortés and F. Bullo (2003). Analysis and design of oscillatory control systems. IEEE Transactions on Automatic Control 48(7), 1164-1177.

Ostrowski, J. P. and J. W. Burdick (1998). The geometric mechanics of undulatory robotic locomotion. International Journal of Robotics Research 17(7), 683-701.

Paramonov, G. K. (1993). Coherent control of linear and nonlinear excitation of molecular vibrations. Chemical Physics 177(1), 169180.

Sanders, J. A. and F. Verhulst (1985). Averaging Methods in Nonlinear Dynamical Systems. Springer Verlag. New York.

Sanders, S. R., J. M. Noworolski, X. Z. Liu and G. C. Verghese (1991). Generalized averaging method for power conversion circuits. IEEE Transactions on Power Electronics 6(2), 251259.

Seifert, A. and L. G. Pack (1999). Oscillatory control of separation at high Reynolds numbers. AIAA Journal 37(9), 1062-71.

Warren, W. S., H. Rabitz and M. Dahleh (1993). Coherent control of quantum dynamics - the dream is alive. Science 259(5101), 1581-1589. 